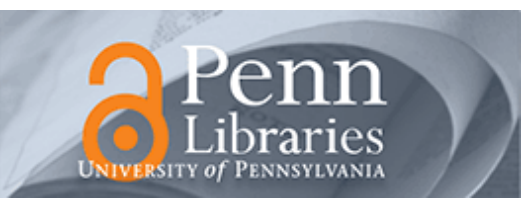

University of Pennsylvania

ScholarlyCommons

\title{
Modeling the Dynamics of Network Technology Adoption and the Role of Converters
}

\author{
Soumya Sen \\ University of Pennsylvania, soumyas@princeton.edu \\ Youngmi Jin \\ University of Pennsylvania, jinnie00@gmail.com \\ Roch A. Guérin \\ University of Pennsylvania, guerin@acm.org \\ Kartik Hosanagar \\ University of Pennsylvania, kartikh@wharton.upenn.edu \\ Follow this and additional works at: https://repository.upenn.edu/ese_papers \\ Part of the Digital Communications and Networking Commons, Management Sciences and \\ Quantitative Methods Commons, and the Systems and Communications Commons
}

\section{Recommended Citation}

Soumya Sen, Youngmi Jin, Roch A. Guérin, and Kartik Hosanagar, "Modeling the Dynamics of Network Technology Adoption and the Role of Converters", . April 2010.

Copyright 2010 IEEE. Preprint version.

Accepted for IEEE/ACM Transactions on Networking. Postprint version will be made available at the publisher URL:

http://dx.doi.org/10.1109/TNET.2010.2048923

This material is posted here with permission of the IEEE. Such permission of the IEEE does not in any way imply IEEE endorsement of any of the University of Pennsylvania's products or services. Internal or personal use of this material is permitted. However, permission to reprint/republish this material for advertising or promotional purposes or for creating new collective works for resale or redistribution must be obtained from the IEEE by writing to pubs-permissions@ieee.org. By choosing to view this document, you agree to all provisions of the copyright laws protecting it.

This paper is posted at ScholarlyCommons. https://repository.upenn.edu/ese_papers/531

For more information, please contact repository@pobox.upenn.edu. 


\title{
Modeling the Dynamics of Network Technology Adoption and the Role of Converters
}

\begin{abstract}
New network technologies constantly seek to displace incumbents. Their success depends on technological superiority, the size of the incumbent's installed base, users' adoption behaviors, and various other factors. The goal of this paper is to develop an understanding of competition between network technologies, and identify the extent to which different factors, in particular converters (a.k.a. gateways), affect the outcome. Converters can help entrants overcome the influence of the incumbent's installed base by enabling cross-technology interoperability. However, they have development, deployment, and operations costs, and can introduce performance degradations and functionality limitations, so that if, when, why, and how they help is often unclear. To this end, the paper proposes and solves a model for adoption of competing network technologies by individual users. The model incorporates a simple utility function that captures key aspects of users' adoption decisions. Its solution reveals a number of interesting and at times unexpected behaviors, including the possibility for converters to reduce overall market penetration of the technologies and to prevent convergence to a stable state; something that never arises in their absence. The findings were tested for robustness, e.g., different utility functions and adoption models, and found to remain valid across a broad range of scenarios.
\end{abstract}

\section{Keywords}

Technology adoption, diffusion, externality, converters, dynamics, equilibrium.

\section{Disciplines}

Digital Communications and Networking | Management Sciences and Quantitative Methods | Systems and Communications

\section{Comments}

Copyright 2010 IEEE. Preprint version.

Accepted for IEEE/ACM Transactions on Networking. Postprint version will be made available at the publisher URL:

http://dx.doi.org/10.1109/TNET.2010.2048923

This material is posted here with permission of the IEEE. Such permission of the IEEE does not in any way imply IEEE endorsement of any of the University of Pennsylvania's products or services. Internal or personal use of this material is permitted. However, permission to reprint/republish this material for advertising or promotional purposes or for creating new collective works for resale or redistribution must be obtained from the IEEE by writing to pubs-permissions@ieee.org. By choosing to view this document, you agree to all provisions of the copyright laws protecting it. 


\title{
Modeling the Dynamics of Network Technology Adoption and the Role of Converters
}

\author{
Soumya Sen, Student Member, IEEE Youngmi Jin, Member, IEEE \\ Roch Guérin, Fellow, ACM; Fellow IEEE and Kartik Hosanagar
}

\begin{abstract}
New network technologies constantly seek to displace incumbents. Their success depends on technological superiority, the size of the incumbent's installed base, users' adoption behaviors, and various other factors. The goal of this paper is to develop an understanding of competition between network technologies, and identify the extent to which different factors, in particular converters (a.k.a. gateways), affect the outcome. Converters can help entrants overcome the influence of the incumbent's installed base by enabling cross-technology interoperability. However, they have development, deployment, and operations costs, and can introduce performance degradations and functionality limitations, so that if, when, why, and how they help is often unclear. To this end, the paper proposes and solves a model for adoption of competing network technologies by individual users. The model incorporates a simple utility function that captures key aspects of users' adoption decisions. Its solution reveals a number of interesting and at times unexpected behaviors, including the possibility for converters to reduce overall market penetration of the technologies and to prevent convergence to a stable state; something that never arises in their absence. The findings were tested for robustness, e.g., different utility functions and adoption models, and found to remain valid across a broad range of scenarios.
\end{abstract}

Index Terms-Technology adoption, diffusion, externality, converters, dynamics, equilibrium.

\section{INTRODUCTION}

Advances in technology often see newer and better solutions replacing older ones. Networking is no exception. For example, the Internet competed against alternative packet data technologies before finally displacing the phone network as the de facto communication infrastructure. Recently, there have been calls for new architectures to succeed it, and these will face a formidable incumbent in the Internet. Their eventual success in replacing it will likely depend not just on technical superiority, but also on economic factors, and on their ability to win over the Internet's installed base.

A large installed base can give an incumbent an edge even if a new (entrant) technology is technically superior. The traditional networking approach to this problem has been converters (a.k.a. gateways) to ease migration from one

A preliminary version of this work was presented at the NetEcon'08 Workshop

S. Sen, Y. Jin and R. Guérin are with the Department of Electrical and Systems Engineering, University of Pennsylvania, PA, 19104, USA, email: (guerin@ee.upenn.edu). Kartik Hosanagar is with the Operations and Information Management Department at the Wharton School, University of Pennsylvania.

Manuscript received 22 June, 2009; revised 24 December 2009; Accepted 9 April 2010.

This work was supported by NSF grant CNS-0721610 technology to another. This is not unique to networks, but converters are particularly important in network settings where "communication" is the primary function, and its benefits grow with the number of users that can be reached, e.g., as in Metcalfe's Law. Since converters allow users of one technology to connect with users of another, they are an important tool in the adoption of network technologies. However, developing, deploying, and operating converters comes at a cost, one that often grows as a function of the converter's quality. Further, converters can play a directionally ambiguous role. On one hand, a converter can help the entrant overcome the advantage of the incumbent's large installed base by allowing connectivity to it. On the other hand, the converter also helps the incumbent technology by mitigating the impact of its users migrating to the newer technology. Understanding the impact of converters on network technology adoption is, therefore, a topic that deserves further scrutiny.

In this paper, we develop a modeling framework to study adoption dynamics of entrant and incumbent technologies in the presence of network externalities. Specifically, we introduce a model for the utility derived by an individual user from a communication network, and use it to build an aggregate model for technology adoption that is consistent with individual rational decision-making. We apply the model to study the role that converters can play in the adoption of network technologies. Our main findings are:

- The adoption process can exhibit multiple steady state outcomes (equilibria); each associated with a specific region of initial adoption levels for the two technologies. For example, an entrant technology may succeed only if the incumbent is not already well entrenched.

- Converters can help a technology improve its own standing, i.e., market share, and even ensure its dominance while it would have entirely disappeared in the absence of converters. For example, a low-quality but low-cost technology may thwart the success of a better but more expensive competitor by preserving the ability of its users to access adopters of the pricier technology, whose usage would then be limited to a few "techno-buffs."

- Improving converters' efficiency can at times be harmful. They can result in lower market shares for an individual technology or for both. For instance, high market penetration may depend on the combination of a cheap but lowend technology with a high-end but more expensive one to adequately serve the full spectrum of user preferences. A situation where converters allow the better technology 
to gain market share at the expense of the lesser technology may result in low-end users of that technology dropping out altogether; thereby contributing to a lower overall market penetration.

- While in the absence of converters technology adoption always converges to a stable steady-state equilibrium, this need not be so when converters are present. Boom-andbust cycles in which users switch back-and-forth between technologies can arise when technologies are asymmetric in the externality benefits they offer.

The rest of this paper is organized as follows: Section II introduces our model and problem formulation. Section III characterizes technology adoption trajectories and equilibrium adoption levels. Section IV explores the role of converters in influencing adoption outcomes. Section V reviews prior work and positions the paper in the literature. We discuss the limitations of this study and conclude the paper with remarks on future work in Section VI. The proofs of all propositions can be found in [15].

\section{Technology Adoption Model}

\section{A. Technology Valuation}

As in most competitive situations, the choice of one technology over another depends on the "value" they provide. Value is a somewhat elusive concept that depends in part on the quality and functionality of the technology and its cost. In the context of network technologies whose main purpose is to enable communication among users, the number of users ${ }^{1}$ accessible through it is another important component, often termed network effect or externality. As commonly done, we account for these factors and their effect on technology adoption through a utility function. For two competing network technologies, 1 and 2, the respective utility functions are given by eqs. (1) and (2).

$$
\begin{aligned}
& U_{1}=\theta q_{1}+\left(x_{1}+\alpha_{1} \beta x_{2}\right)-p_{1} \\
& U_{2}=\theta q_{2}+\left(\beta x_{2}+\alpha_{2} x_{1}\right)-p_{2}
\end{aligned}
$$

Eqs. (1) and (2) consist of three distinct terms. Focusing on, say, Technology 1 , the first term, $\theta q_{1}$ represents the standalone benefits from the technology, with $q_{1}$ representing the intrinsic quality of the technology, and $\theta$ a random variable accounting for heterogeneity in how users value technology. The quantity $q_{1}$ incorporates aspects of functionality, reliability, performance, etc., for the technology. In the rest of the paper, we assume $q_{2}>q_{1}$, i.e., Technology 2 is superior to Technology 1 and correspondingly can be viewed as the entrant with Technology 1 playing the role of the incumbent. The model, however, does not mandate such an assignment of roles, e.g., it can be used to study settings where Technology 1 is the entrant and offers, say, a lower quality but cheaper alternative than the incumbent Technology 2. The random variable $\theta \in[0,1]$ determines the relative weight a user places on the intrinsic quality of a technology. It is private information, but we assume that the distribution of $\theta$ across

${ }^{1}$ Users can be individuals or organizations, and include resources and content. users is known. We make the common assumption [2] that $\theta$ is uniformly distributed in the interval $[0,1]$. This choice affects the magnitude of equilibrium adoption levels, but does not qualitatively affect findings regarding technology adoption dynamics and outcomes as demonstrated in [15, Appendix F].

The second component of the user's utility is the network externality (or network effect), which refers to benefits derived from the ability to connect with other users. Network externalities are chosen to be proportional to the number of users to which each technology gives access. This linear dependency of network benefits on the number of adopters is consistent with Metcalfe's Law and commonly used in the literature [7]. In [15, Appendix F], we investigate other models and demonstrate the robustness of our findings across different functional forms for network externality, including non-linear ones. Denoting as $x_{1}$ and $x_{2}$ the fractions of adopters of each technology out of a large population of size $N$, the externality benefits for Technology 1 consist of $x_{1}$, the fraction of Technology 1 users, plus $\alpha_{1} \beta x_{2}$, a term that includes the fraction of Technology 2 users weighed by two additional factors. The first, $0 \leq \alpha_{1} \leq 1$, captures the availability of converters offering connectivity from Technology 1 to Technology 2 ( $\alpha_{1}=0$ corresponds to no converter and $\alpha_{1}=1$ to "perfect" converters). The second parameter, $\beta$, allows different externality benefits for the two technologies ${ }^{2}$. We note that converters, once deployed, are available to all users of the technology. This corresponds to what we term "technology-level" converters, i.e., their development and deployment are decisions made by the providers of network technologies.

Converters can be characterized as either duplex or simplex, symmetric or asymmetric, and constrained or unconstrained. Duplex converters provide bi-directional connectivity between technologies, while simplex converters are present in only one direction (most network technologies involve duplex converters, but the model does not mandate them). Asymmetric converters simply refer to the fact that converter efficiency can be different in each direction i.e., $\alpha_{1} \neq \alpha_{2}$. The notion of constrained vs. unconstrained converters arises in the presence of technologies that exhibit different externalities, i.e., $\beta \neq 1$. For example, when $\beta>1$, it captures whether converters allow users of Technology 1 access to the greater externality benefits of Technology 2 when connecting to its users. A converter is unconstrained if this is permitted, i.e., $\alpha_{1} \beta>1$. We discuss an example where this can arise at the end of the section.

The last element of eqs. (1) and (2) is the price, $p_{i}, i \in$ $\{1,2\}$. Because of our focus on networks and connectivity that is typically offered as a service rather than a good or product, price is recurrent. In other words, maintaining connectivity through a particular network technology incurs new charges at regular intervals. As a result, users continuously reevaluate their technology choices, and can switch from one technology to another and possibly back. For analytical tractability, the model assumes that switching costs are negligible. This represents a reasonable assumption in many settings. For example,

\footnotetext{
${ }^{2}$ Eqs. (1) and (2) implicitly express utility in units of Technology 1 externality benefits, i.e., Technology 1 externality benefits are equal to 1 when its penetration level is $100 \%\left(x_{1}=1\right)$.
} 
the very high customer churn (reported to range from $72 \%$ all the way up to $98 \%$ per year [4], [13]) that prevails in the ISP market points to little or no switching costs in that market. On the other hand, non-zero switching costs, in the form of contract breaking penalties and learning costs, are the norm in many settings. A natural question is, therefore, whether behaviors observed in the absence of switching costs hold when they are present. Extending the analytical model to incorporate switching costs is challenging, especially if one is to consider the many possible types of switching costs, but numerical investigations are feasible. Appendix G of [15] reports on these investigations, which demonstrate a relative insensitivity to switching costs. Obviously, as switching costs increase, they eventually eliminate all adoption dynamics, but the different behaviors that the (simple) model of the paper helped reveal persist over a non-trivial range.

We note that the model parameters, i.e., $q_{i}, p_{i}, \alpha_{i}, \beta$, are static and exogenously specified. An obvious extension is to make them time-varying, e.g., technology gets better and/or cheaper as time goes by, and the outcome of strategic decisionmaking. Developing game-theoretic models that incorporate such effects is clearly of interest, especially in the context of competitive scenarios where firms may offer introductory pricing or seed the market to gain an initial foothold. Exploring those issues, however, requires that we first understand the basic tenets of technology adoption and dynamics in the simpler setting considered in this paper.

Another important question is how to assign actual values to the model's parameters. This is a topic that goes well beyond this paper, and we only point to a possible approach. A common method to estimate utility weights is conjoint analysis, a technique that has been widely adopted by marketing researchers and practitioners (see [9] for a detailed review). It relies on surveys offering users different combinations of functionality and attributes to extract a relative ordering among them, and ultimately produce individual weight assignments.

\section{B. User Decisions}

Given current adoption levels, $x_{1}$ and $x_{2}$, the utility functions of eqs. (1) and (2) identify how a user values each technology, which in turn determines her technology selection decisions. Specifically, a user chooses Technology $i$ whenever it provides a surplus that is both positive (Individual Rationality constraint) and higher than that of the other technology (Incentive Compatibility constraint). In other words, a user chooses

$$
\left\{\begin{array}{lll}
\text { no technology } & \text { if } & U_{i}<0 \text { for all } i, \\
\text { Technology } 1 & \text { if } & U_{1}>0 \text { and } U_{1}>U_{2}, \\
\text { Technology } 2 & \text { if } & U_{2}>0 \text { and } U_{2}>U_{1} .
\end{array}\right.
$$

Note that the model assumes an exclusive choice of technology by users, i.e., they select Technology 1 , or 2 , or neither, but not both. This translates into the constraint $0 \leq$ $x_{1}+x_{2} \leq 1$. The dynamics of technology adoption arise from the dependency of the $U_{i}$ 's on the $x_{i}$ 's that change with users' adoption decisions. Capturing these dynamics, therefore, calls for specifying when users become aware of changes in the $x_{i}$ 's and update their adoption decisions. Knowledge of changes in adoption levels is likely to diffuse through the user population and users' reactions are often heterogeneous, i.e., some switch quickly, while others defer. An approach, commonly used in individual-level diffusion models [10] and that captures these aspects is a continuous time approximation. Specifically, assume that at time $t$ the "current" technology adoption levels, $\underline{x}(t)=\left(x_{1}(t), x_{2}(t)\right)$, are known to all users. With this information, users can compute their utility for each technology and make adoption decisions. Let $H_{i}(\underline{x}(t)), i \in\{1,2\}$ denote the fraction of users for whom Technology $i$ provides the highest (and positive) utility ${ }^{3}$. The quantity $H_{i}(\underline{x}(t))-x_{i}(t)$ corresponds to the fraction of users that would normally proceed to adopt (disadopt) Technology $i$ at time $t$. To capture a progressive adoption process, we assume that the rate of change in users' technology choices is proportional to this quantity, namely,

$$
\frac{d x_{i}(t)}{d t}=\gamma\left(H_{i}(\underline{x}(t))-x_{i}(t)\right), i \in\{1,2\},
$$

The quantity $\gamma<1$ is analogous to the hazard rate in diffusion models, and can be viewed as the expected conditional probability that an individual who has not yet adopted technology $i$ will do so at time $t$. In our analysis, we assume that the propensity of individuals to adopt does not change with time, i.e., $\gamma$ is constant.

Two aspects of this diffusion process need further clarification. First, users are myopic. At any instant, the adoption decisions are driven by the number of adopters at that time $\left(x_{i}(t)\right)$ and users are not able to anticipate likely adoption levels in the future. This is analogous to a best response dynamic. Second, the model identifies the rate of technology adoption across users, but not which users are making the change. To preserve consistency with user preferences, $\theta$, we assume that the first users to adopt Technology $i$ are those that stand to benefit most from the action. This ensures that at all times the sets of users having adopted either technology correspond to blocks of users with contiguous technology preferences.

The diffusion dynamics governed by eq. (3) can converge to a steady-state equilibrium $\underline{x}^{*}$ characterized by:

$$
\left.\frac{d x_{i}(t)}{d t}\right|_{\underline{x}(t)=\underline{x}^{*}} ^{=0} \Leftrightarrow x_{i}^{*}=H_{i}\left(\underline{x}^{*}\right) \text { for } i \in\{1,2\} .
$$

In other words, at equilibrium, the fraction of users for whom it is individually rational and incentive compatible to choose Technology $i$ equals the current fraction of adopters of Technology $i$. Based on this formulation, our goal is to characterize, as a function of the exogenous system parameters $\beta, p_{i}, q_{i}, \alpha_{i}$ for $i \in\{1,2\}$, the equilibrium adoption levels, i.e., the fixed points of eq. (4), and the dynamics leading to them.

Before exploring the dynamics and equilibria of technology adoption that the model gives rise to, we pause to briefly introduce a couple of examples that illustrate the model's parameters and applicability.

IPv4 vs. IPv6: The impending exhaustion of IPv4 addresses, e.g., http:/www.potaroo.net/tools/ipv4 for a daily countdown,

\footnotetext{
${ }^{3}$ We discuss the derivation of $H_{i}(\underline{x}(t))$ in Section III-A.
} 
implies that service providers signing up new Internet customers will have to start using IPv6 addresses or charge more users who insist on an IPv4 address, i.e., $p_{\mathrm{IPv} 4}=p_{1}>$ $p_{2}=p_{\text {IPv6 }}$. As technologies, although IPv4 and IPv6 are incompatible, they are largely similar so that for the purpose of our model one can reasonably assume $q_{1} \lesssim q_{2}$ and $\beta=1$. Because of their incompatibility, converters (gateways), e.g., see [5] for a representative recent proposal, are needed for IPv6 users to access the IPv4 content that is the bulk of today's Internet content and unlikely to become natively accessible over IPv6 any time soon ${ }^{4}$. Conversely, those converters also enable the reverse flow from IPv4 to IPv6, i.e., they are duplex converters, albeit not necessarily delivering the same performance in both directions, i.e., they can be asymmetric, so that both $\alpha_{1}$ and $\alpha_{2}$ are non-zero but not always equal.

Users then decide between subscribing to an IPv4 or IPv6 service on the basis of price $\left(p_{i}\right)$, the level of content they are able to access $\left(x_{i}\right)$, and the quality of that access $\left(\alpha_{i}\right)$.

Low Def. vs. High Def. Video: The previous example illustrated a common adoption scenario with two mostly equivalent technologies and duplex, asymmetric converters. Because of the similarity of the two technologies $(\beta=1)$, converters were by default constrained $\left(\alpha_{1} \beta \leq 1\right)$. However, when technologies exhibit significant differences in externality benefits, e.g., $\beta>1$, converters can be unconstrained $\left(\alpha_{1} \beta>1\right)$ and we present next a possible example.

Consider a provider that offers its customers a videoconferencing service with the associated equipment. The service comes in two versions, high-definition (HD) and standarddefinition (SD), i.e., HD equipment generates a high-quality $\left(q_{2}\right)$ video signal while SD equipment produces a lower resolution $\left(q_{1}<q_{2}\right)$. Users derive value from video-conferencing with one another, with $\beta>1$ reflecting the higher quality of an HD signal. The two services are priced accordingly $\left(p_{2}>p_{1}\right)$. However, because video is a highly asymmetric technology (encoding is hard but decoding is comparatively easy), it is possible for the provider to enable the decoding of HD signal on SD equipment (and obviously conversely). This conversion can introduce quality degradations $\left(\alpha_{1}<1\right)$, but more importantly it allows SD users access to the externality benefits associated with receiving HD signals. Assuming $\mathrm{HD} \leftrightarrow \mathrm{SD}$ conversion is available in both direction, this is an instance of a duplex, possibly asymmetric $\left(\alpha_{1} \neq \alpha_{2}\right)$, and unconstrained $\left(\alpha_{1} \beta>1\right)$ converter.

Many users may then opt for the SD service because of its lower price and the ability to still enjoy the higher benefits of viewing HD signals. On the other hand, if all users were to select the SD service, those externality benefits would disappear. In general, users with high technology valuation $(\theta$ close to 1) may still opt for the HD service, but the decision depends on choices made by others.

\section{TRAJECTORIES AND EQUILIBRIA}

Solving the evolution of technology adoption decisions over time described in eq. (3) calls for first computing expressions

\footnotetext{
${ }^{4}$ Although the servers hosting most web sites can typically get an IPv6 address, very few have bothered registering one with DNS, e.g., see http://bgp.he.net/ipv6-progress-report.cgi.
}

for $H_{i}(\underline{x}(t)), i=\{1,2\}$ as functions of known parameters.

\section{A. Characterizing $H_{i}(\underline{x})$}

For notational convenience we omit dependency on time and write $\underline{x}(t)$ simply as $\underline{x}$. Recall that $H_{i}(\underline{x}), i \in\{1,2\}$, corresponds to the fraction of users for whom it is rational to adopt Technology $i$, given the current adoption levels, $\underline{x}$. To determine the fraction of adopters of each technology, we introduce the notion of indifference points, which identify thresholds in users technology valuation $(\theta)$ corresponding to changes in technology preference. Specifically, $\theta_{i}^{0}(\underline{x}), i \in$ $\{1,2\}$ identify the $\theta$ value separating users with a negative utility for Technology $i$ from those with a positive utility. In other words, for technology penetration levels $\underline{x}, \theta_{i}^{0}(\underline{x})$ is such that $U_{i}\left(\theta_{i}^{0}, \underline{x}\right)=0$, and $U_{i}(\theta, \underline{x})$ is positive (negative) for $\theta$ values larger (smaller) than $\theta_{i}^{0}$.

From eqs. (1) and (2), $U_{i}\left(\theta_{i}^{0}, \underline{x}\right)=0$ gives

$$
\begin{aligned}
& \theta_{1}^{0}(\underline{x})=\frac{p_{1}-\left(x_{1}+\alpha_{1} \beta x_{2}\right)}{q_{1}} \\
& \theta_{2}^{0}(\underline{x})=\frac{p_{2}-\left(\beta x_{2}+\alpha_{2} x_{1}\right)}{q_{2}}
\end{aligned}
$$

Similarly, $\theta_{2}^{1}(\underline{x})$ corresponds to the $\theta$ value separating users preferring Technology 1 from those preferring Technology 2, i.e., $U_{1}\left(\theta_{2}^{1}, \underline{x}\right)=U_{2}\left(\theta_{2}^{1}, \underline{x}\right)$ and users with $\theta>\theta_{2}^{1}(\underline{x})$ derive greater utility from Technology 2 than Technology 1 (recall that $\left.q_{2}>q_{1}\right)$. Setting, $U_{1}\left(\theta_{2}^{1}, \underline{x}\right)=U_{2}\left(\theta_{2}^{1}, \underline{x}\right)$ gives

$$
\theta_{2}^{1}(\underline{x})=\frac{\left(1-\alpha_{2}\right) x_{1}-\beta\left(1-\alpha_{1}\right) x_{2}+p_{2}-p_{1}}{q_{2}-q_{1}}
$$

Combining eqs. (5)-(7) gives

$$
\begin{aligned}
& \theta_{2}^{1}(\underline{x})-\theta_{1}^{0}(\underline{x})=\frac{q_{2}}{q_{2}-q_{1}}\left(\theta_{2}^{0}(\underline{x})-\theta_{1}^{0}(\underline{x})\right), \\
& \theta_{2}^{1}(\underline{x})-\theta_{2}^{0}(\underline{x})=\frac{q_{1}}{q_{2}-q_{1}}\left(\theta_{2}^{0}(\underline{x})-\theta_{1}^{0}(\underline{x})\right),
\end{aligned}
$$

from which the following Proposition can be derived.

\section{Proposition 1:}

If $\theta_{1}^{0}(\underline{x})<\theta_{2}^{0}(\underline{x})$, then $\theta_{2}^{1}(\underline{x})>\theta_{2}^{0}(\underline{x})>\theta_{1}^{0}(\underline{x})$. If $\theta_{1}^{0}(\underline{x}) \geq \theta_{2}^{0}(\underline{x})$, then $\theta_{2}^{1}(\underline{x}) \leq \theta_{2}^{0}(\underline{x}) \leq \theta_{1}^{0}(\underline{x})$.

Proposition 1 constrains the possible orderings of the indifference points given by eqs. (5)-(7), so that $H_{i}(\underline{x}), i \in\{1,2\}$ can be characterized in a compact manner.

$H_{1}(\underline{x})= \begin{cases}{\left[\theta_{2}^{1}(\underline{x})\right]_{[0,1]}-\left[\theta_{1}^{0}(\underline{x})\right]_{[0,1]}} & \text { if } \theta_{1}^{0}(\underline{x})<\theta_{2}^{0}(\underline{x}) \\ 0 & \text { otherwise }\end{cases}$

$H_{2}(\underline{x})= \begin{cases}1-\left[\theta_{2}^{1}(\underline{x})\right]_{[0,1]} & \text { if } \theta_{1}^{0}(\underline{x})<\theta_{2}^{0}(\underline{x}) \\ 1-\left[\theta_{2}^{0}(\underline{x})\right]_{[0,1]} & \text { otherwise }\end{cases}$

where $y_{[a, b]}$ is the 'projection' 5 ' of $y$ into $[a, b]$.

The expressions for $H_{1}(\underline{x})$ and $H_{2}(\underline{x})$ determine the trajectory as well as the equilibrium outcome of the adoption process as per eqs. (3) and (4) respectively. Eq. (10) characterizes $H_{i}(\underline{x}), i=\{1,2\}$ through multiple possible expressions that depend on the relative ordering of $\theta_{1}^{0}(\underline{x}), \theta_{2}^{0}(\underline{x})$ and $\theta_{2}^{1}(\underline{x})$,

\footnotetext{
${ }^{5}$ i.e., its value is $y$ for $y \in[a, b], a$ for $y<a$, and $b$ for $y>b$.
} 
and the outcome of their projections on $[0,1]$. Identifying the different combinations that eq. (10) gives rise to calls for partitioning the $\left(x_{1}, x_{2}\right)$-plane into regions, each corresponding to unique expressions for the $\left(H_{1}(\underline{x}), H_{2}(\underline{x})\right)$ pair. A method for constructing such a partition is described next.

First, consider all values of $\underline{x}$ which satisfy $\theta_{1}^{0}(\underline{x}) \geq \theta_{2}^{0}(\underline{x})$. In the corresponding half-plane of the $\left(x_{1}, x_{2}\right)$-plane, $H_{1}(\underline{x})$ is always 0 , but the value of $H_{2}(\underline{x})$ depends on the projection of $\theta_{2}^{0}(\underline{x})$ on $[0,1]$ (i.e., whether $\theta_{2}^{0}(\underline{x}) \leq 0,0<\theta_{2}^{0}(\underline{x})<1$, or $1 \leq$ $\left.\theta_{2}^{0}(\underline{x})\right)$. This creates three regions in the $\left(x_{1}, x_{2}\right)$-plane, each with a different expression for the $\left(H_{1}(\underline{x}), H_{2}(\underline{x})\right)$ pair. These three regions, labeled $R_{1}, R_{2}$ and $R_{3}$, and the corresponding conditions on $\theta_{2}^{0}(\underline{x})$ appear in the left side of Table I. The expressions for $H_{i}(\underline{x}), i=\{1,2\}$ in each region are provided in Table II.

Conversely, for values of $\underline{x}$ which satisfy $\theta_{1}^{0}(\underline{x})<\theta_{2}^{0}(\underline{x})$ (the other half-plane), eq. (10) indicates that expressions for $H_{1}(\underline{x})$ and $H_{2}(\underline{x})$ depend on the positions of both $\theta_{2}^{1}(\underline{x})$ and $\theta_{1}^{0}(\underline{x})$ relative to 0 and 1 (i.e., whether $\theta_{2}^{1}(\underline{x}) \leq 0,0<\theta_{2}^{1}(\underline{x})<1$ or $1 \leq \theta_{2}^{1}(\underline{x})$, and similarly for $\left.\theta_{1}^{0}(\underline{x})\right)$. This yields nine possible combinations. The number of feasible combinations can, however, be reduced to six using Proposition 1, which constrains $\theta_{1}^{0}(\underline{x})<\theta_{2}^{0}(\underline{x})<\theta_{2}^{1}(\underline{x})$. For example, when $\theta_{1}^{0}(\underline{x})<\theta_{2}^{0}(\underline{x}), \theta_{2}^{1}(\underline{x}) \leq 0$ and $1 \leq \theta_{1}^{0}(\underline{x})$ is infeasible per Proposition 1. Thus in the half-plane $\theta_{1}^{0}(\underline{x})<\theta_{2}^{0}(\underline{x})$, there are six possible expressions for $H_{i}(\underline{x}), i=\{1,2\}$. These expressions are reported on the right side of Table I, with the corresponding regions labeled $R_{4}$ to $R_{9}$. Combining the two half-planes gives a total of nine regions, $R_{1}$ to $R_{9}$, where in each region the $\left(H_{1}(\underline{x}), H_{2}(\underline{x})\right)$ pair has a unique expression as specified in Table II.

This partitioning in nine regions has a graphical representation, as shown in Figure 1. The line $\theta_{1}^{0}(\underline{x})=\theta_{2}^{0}(\underline{x})$ splits the $\left(x_{1}, x_{2}\right)$-plane in the two previously mentioned half-planes. The two lines corresponding to $\theta_{2}^{0}(\underline{x})=0$ and $\theta_{2}^{0}(\underline{x})=1$ are parallel, and define the three regions $R_{1}, R_{2}$ and $R_{3}$ in the half-plane $\theta_{1}^{0}(\underline{x}) \geq \theta_{2}^{0}(\underline{x})$. Similarly, the lines $\theta_{1}^{0}(\underline{x})=0$, $\theta_{1}^{0}(\underline{x})=1, \theta_{2}^{1}(\underline{x})=0$ and $\theta_{2}^{1}=1$, divide the second half-plane into the six regions, $R_{4}$ to $R_{9}$. Figure 1 also illustrates that the lines $\theta_{2}^{0}(\underline{x})=0, \theta_{1}^{0}(\underline{x})=0$ and $\theta_{2}^{1}(\underline{x})=0$ always intersect at a point denoted as $P$, and the lines $\theta_{2}^{0}(\underline{x})=1, \theta_{1}^{0}(\underline{x})=1$ and $\theta_{2}^{1}(\underline{x})=1$ always intersect at a point denoted as $Q$, with both $P$ and $Q^{6}$ lying on the line $\theta_{1}^{0}=\theta_{2}^{0}$. The points $P$ and $Q$ can be shown to act as "anchors" that ensure that the $\left(x_{1}, x_{2}\right)$ plane is always partitioned into exactly nine regions with fixed relative positions.

It should also be noted that all nine regions need not always be feasible. Whether or not they are feasible depends on their relative position in the solution space, $S=\left\{\left(x_{1}, x_{2}\right)\right.$ s.t. $0 \leq$ $\left.x_{1} \leq 1,0 \leq x_{2} \leq 1, x_{1}+x_{2} \leq 1\right\}$. The number of regions that lie inside $S$ (and hence are relevant to the analysis) is a function of the system parameters $\left(q_{i}, p_{i}, \beta, \alpha_{i} ; i=\{1,2\}\right)$. Last but not least, as shown in [15, Appendix B], the partitioning of the solution space into nine regions actually holds for more generic (monotonic) externality functions, i.e., it is

\footnotetext{
${ }^{6}$ The coordinates of the points $P$ and $Q$ can be readily found by solving simple systems of linear equations given by eqs.(5)-(7).
}

not an artifact of the simplified linear externality function.

Finally, we pause to briefly interpret the conditions that define each region, and their implications for solutions. We do so by way of an example, focusing on Region $R_{8}$. Region $R_{8}$ is defined as the set of adoption levels, $\underline{x}=\left(x_{1}, x_{2}\right)$, for which $1 \leq \theta_{2}^{1}(\underline{x})$ and $0 \leq \theta_{1}^{0}(\underline{x})<1$. The condition $1 \leq \theta_{2}^{1}(\underline{x})$ implies that in Region 8 all users prefer Technology 1 over 2 . Hence any existing Technology 2 adopter will disadopt. Thus, in $R_{8}$, users can either be non-adopters of both technologies $\left(0<\theta<\theta_{1}^{0}(\underline{x})\right)$ or adopters of Technology 1 (if $\theta>\theta_{1}^{0}(\underline{x})$ ). Similar interpretations are possible for other regions.

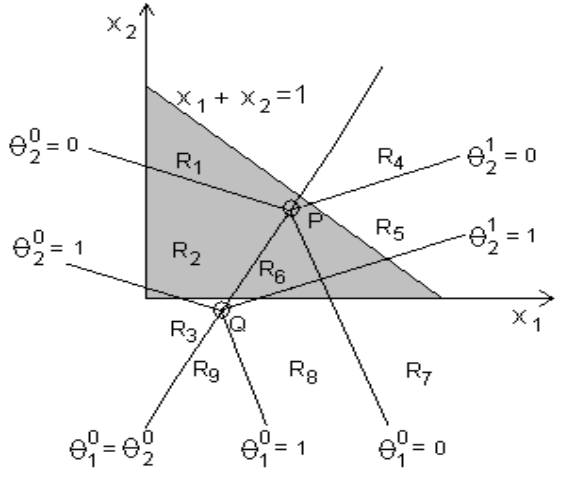

Fig. 1. Region Partitions

\section{B. Characterizing Adoption Trajectories}

By combining eqs. (5) to (7) with eq. (10), explicit expressions can be obtained for $H_{i}(\underline{x})$ in each of the nine regions. These are listed in Table II. Using these expressions, it is now possible to solve eq. (3) and characterize the trajectory of technology adoption in each region. The trajectories have the following general form:

$$
x_{i}(t)=a_{i}+b_{i} e^{\lambda_{1} t}+c_{i} e^{\lambda_{2} t}, \quad i \in\{1,2\}
$$

where $\lambda_{1}$ and $\lambda_{2}$ can be positive, negative, or complex depending on the region. Individual solutions for each region are listed in Table III of the Appendix.

The full trajectory of technology adoption starting at some initial adoption levels $\underline{x}(0)$ within a given region, can then be obtained by "stitching" together trajectories in individual regions as region boundaries are crossed. The next question is to determine whether and where these trajectories may eventually converge as $t \rightarrow \infty$. We tackle this issue next.

\section{Computing Steady-state Equilibria}

From eq. (11), we see that a technology adoption trajectory in, say, region $R_{k}$, converges to a stable equilibrium $x_{i}(\infty)=$ $a_{i}, i \in\{1,2\}$, if $\lambda_{1}$ and $\lambda_{2}$ are both negative (equilibrium is locally stable), and $\left(a_{1}, a_{2}\right) \in S \cap R_{k}$ (the equilibrium is valid, i.e., in the region associated with the trajectory). In other words, solutions to eq. (4) $\left(H_{i}\left(\underline{x}^{*}\right)=x_{i}^{*}, i \in\{1,2\}\right)$, must satisfy stability and validity conditions to be valid steadystate outcomes of the technology adoption process ${ }^{7}$. The

${ }^{7}$ Our model is well-behaved and instances of boundary fixed points do not arise 
TABLE I

PARTITIONS CHARACTERIZING $H_{i}(\underline{x})$

\begin{tabular}{|c|c|c|cc|}
\hline \multicolumn{2}{|c|}{$\theta_{1}^{0}(\underline{x}) \geq \theta_{2}^{0}(\underline{x})$} & \multicolumn{2}{|c|}{$\theta_{1}^{0}(\underline{x})<\theta_{2}^{0}(\underline{x})$} \\
\hline Region & condition & Region & \multicolumn{2}{|c|}{ condition } \\
\hline$R_{1}$ & $\theta_{2}^{0}(\underline{x}) \leq 0$ & $R_{4}$ & $\theta_{2}^{1}(\underline{x}) \leq 0$, & $0 \leq \theta_{1}^{0}(\underline{x})$ \\
$R_{2}$ & $0<\theta_{2}^{0}(\underline{x})<1$ & $R_{5}$ & $0<\theta_{2}^{1}(\underline{x})<1$, & $\theta_{1}^{0}(\underline{x}) \leq 0$ \\
$R_{3}$ & $1 \leq \theta_{2}^{0}(\underline{x})$ & $R_{6}$ & $0<\theta_{2}^{1}(\underline{x})<1$, & $0<\theta_{1}^{0}(\underline{x})<1$ \\
& & $R_{7}$ & $1 \leq \theta_{2}^{1}(\underline{x})$, & $\theta_{1}^{0}(\underline{x}) \leq 0$ \\
& & $R_{8}$ & $1 \leq \theta_{2}^{1}(\underline{x})$, & $0<\theta_{1}^{0}(\underline{x})<1$ \\
& & $R_{9}$ & $1 \leq \theta_{2}^{1}(\underline{x})$, & $1 \leq \theta_{1}^{0}(\underline{x})$ \\
\hline
\end{tabular}

TABLE II

EXPRESSIONS FOR $H_{i}(\underline{x})$

\begin{tabular}{|c|c|c|}
\hline $\begin{array}{l}R_{1} \\
R_{2} \\
R_{3} \\
\end{array}$ & $H_{1}(\underline{x})=0$ & $\begin{array}{l}H_{2}(\underline{x})=1 \\
H_{2}(\underline{x})=1-\frac{p_{2}-\left(\beta x_{2}+\alpha_{2} x_{1}\right)}{q_{2}} \\
H_{2}(\underline{x})=0\end{array}$ \\
\hline$R_{4}$ & $H_{1}(\underline{x})=0$ & $H_{2}(\underline{x})=1$ \\
\hline$R_{5}$ & $H_{1}(\underline{x})=\frac{\left(1-\alpha_{2}\right) x_{1}-\beta\left(1-\alpha_{1}\right) x_{2}+p_{2}-p_{1}}{q_{2}-q_{1}}$ & $H_{2}(\underline{x})=1-\frac{\left(1-\alpha_{2}\right) x_{1}-\beta\left(1-\alpha_{1}\right) x_{2}+p_{2}-p_{1}}{q_{2}-q_{1}}$ \\
\hline$R_{6}$ & $\begin{aligned} H_{1}(\underline{x})= & \frac{\left(1-\alpha_{2}\right) x_{1}-\beta\left(1-\alpha_{1}\right) x_{2}+p_{2}-p_{1}}{q_{2}-q_{1}} \\
& -\frac{p_{1}-\left(x_{1}+\beta \alpha_{1} x_{2}\right)}{\alpha_{2}}\end{aligned}$ & $H_{2}(\underline{x})=1-\frac{\left(1-\alpha_{2}\right) x_{1}-\beta\left(1-\alpha_{1}\right) x_{2}+p_{2}-p_{1}}{q_{2}-q_{1}}$ \\
\hline$R_{7}$ & $H_{1}(\underline{x})=1$ & \\
\hline$R_{8}$ & $H_{1}(\underline{x})=1-\frac{p_{1}-\left(x_{1}+\beta \alpha_{1} x_{2}\right)}{q_{1}}$ & $H_{2}(\underline{x})=0$ \\
\hline$R_{9}$ & $H_{1}(\underline{x})=0$ & \\
\hline
\end{tabular}

simple nature of eq. (4) makes characterizing valid and stable solutions relatively straightforward, albeit tedious. The results are listed in Tables IV and V in the Appendix. Table IV of the Appendix gives the stability conditions associated with each equilibrium, along with the joint validity and stability conditions (they are inter-dependent) in the last column.

The derivations are mechanical in nature, but we review the implications and properties of their solutions.

First, possible equilibria include instances where one technology wipes out the other while achieving either full $\left(x_{i}^{*}=1\right)$ or partial $\left(0 \leq x_{i}^{*}<1\right)$ market penetration, and instances where both technologies coexist, again at either full $\left(x_{1}^{*}+x_{2}^{*}=\right.$ $1)$ or partial market penetration $\left(0 \leq x_{1}^{*}+x_{2}^{*}<1\right)$. Instances where both technologies die-out, i.e., $\underline{x}^{*}=(0,0)$, while possible (the equilibrium lies in regions $R_{3}$ or $R_{9}$ ), are absent from Table IV (see Appendix), as we restrict our focus to scenarios where Technology 1 survives in the absence of the Technology 2's introduction. This precludes a $(0,0)$ outcome.

Second, although not explicitly indicated in Table IV (see Appendix), configurations can be found for which the validity and stability conditions of multiple equilibria are simultaneously satisfied. In other words, depending on the initial conditions $\underline{x}(0)$, technology adoption converges to different outcomes. The following proposition identifies the configurations of multiple equilibria that can simultaneously arise for a given set of parameter values.

Proposition 2: The only combination of multiple valid and stable equilibria that can coexist are:

1. $(1,0)$ and $(0,1)$

2. $\left(x_{1_{R_{8}}}^{*}, 0\right)$ and $(0,1)$

3. $\left(x_{1_{R_{8}}}^{*}, 0\right)$ and $\left(0, x_{2_{R_{2}}}^{*}\right)$

4. $(1,0)$ and $\left(0, x_{R_{2}}^{*}\right)$

5. $\left(x_{1_{R_{5}}}^{*}, 1-x_{1_{R_{5}}}^{*}\right)$ and $\left(0, x_{2_{R_{2}}}^{*}\right)$
6. $\left(x_{1_{R_{6}}}^{*}, x_{2_{R_{6}}}^{*}\right)$ and $(0,1)$

7. $\left(x_{1_{R_{6}}}^{*}, x_{2_{R_{6}}}^{*}\right)$ and $(1,0)$

Additionally, no combination of three or more equilibria can coexist as valid and stable equilibria.

The proof of the above proposition is available in [15, Appendix D]. When multiple equilibria arise, the initial market penetration determines the equilibrium to which the adoption process converges. Therefore it is useful to identify the set of all initial market levels, $\underline{x}(0)$, for which the adoption trajectory converges to a particular stable equilibrium. This set is known as the 'Basin of Attraction' of that stable equilibrium. If the stable equilibrium is the only stable equilibrium in the system i.e., globally stable, then the entire region $S$ is its basin of attraction. That is, all starting points lead to the equilibrium. But whenever a pair of stable equilibria coexist, a 'separatrix', demarcating the basins of attraction of the two stable equilibria can be computed. The expressions for the separatrices are provided in [15, Table VI, Appendix E].

Figure 2 provides an illustrative example. The figure, called a phase diagram, shows the path of the diffusion process in the $\left(t, x_{1}, x_{2}\right)$ space projected onto the $\left(x_{1}, x_{2}\right)$ plane. In other words, it plots $x_{1}(t)$ versus $x_{2}(t)$ and is what one would see if one stood high on the time axis and looked down into the $\left(x_{1}, x_{2}\right)$ plane, sometimes referred to as the phase plane. We observe that there are two stable steady-state equilibria (of the form $\left(0, x_{2}^{*}\right)$ and $\left.\left(x_{1}^{*}, 0\right)\right)$ and an unstable equilibrium in $R_{6}$. A separatrix passes through this unstable equilibrium, separating the basins of attraction of the stable equilibria.

The framework developed here can be used in a wide range of situations to model the dynamics of adoption. As an illustration of the useful insights that such a model can offer, we apply our model to studying the role of converters in the adoption of incompatible technologies. We see from Tables IV and V 


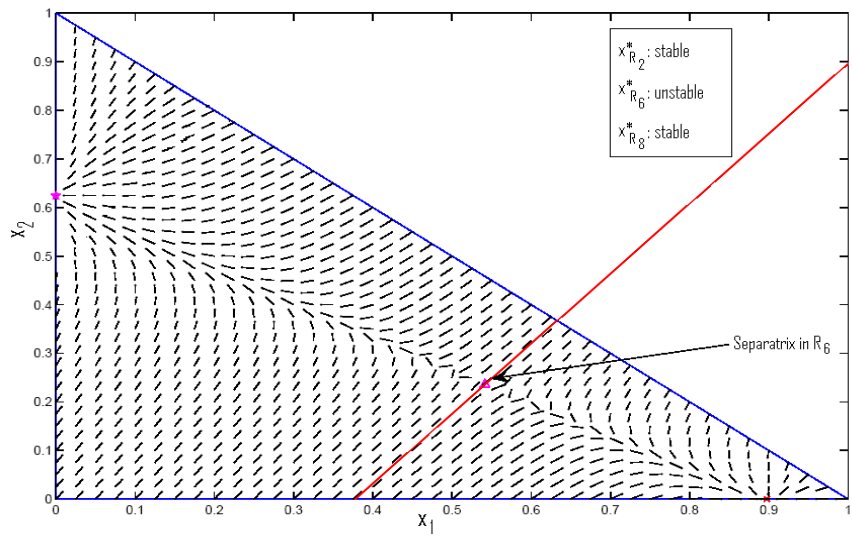

Fig. 2. Separatix and the Basins of Attraction

$\left(p_{1}=1.2, q_{1}=2.95, p_{2}=2.54, q_{2}=5.1, \alpha_{2}=\alpha_{1}=0.01, \beta=1\right)$

of the Appendix that converters can influence (through the parameters $\alpha_{i}$ ) both the validity and the stability of equilibria. In other words, converters may lead technology adoption to an entirely different equilibrium. A rapid inspection of Table III (see Appendix) shows that a similar conclusion holds for trajectories. In particular, converters can affect the values of $\lambda_{1}$ and $\lambda_{2}$ of eq. (11). Investigating if and when such changes can happen, is the topic of Section IV.

\section{THE IMPACT OF CONVERTERS}

As we shall see, converters are capable not just of shifting equilibria around; they can also eliminate or create equilibria. An exhaustive investigation of the full influence of converters, while possible, results in a situation where it is difficult to "see the forest for the trees." As a result, we focus on what we believe are some of the more revealing and significant effects of converters. We identify the reasons behind these effects, and provide conditions under which they can arise.

The investigation proceeds along the following thrusts: (i) Can converters help a network technology improve its market standing and in particular avoid elimination? (ii) Can improving the efficiency of one's converter hurt a technology? (iii) Can improving the efficiency of one's converter hurt the overall market? and (iv) Can the introduction of converters affect overall market stability? Note that when referring to converters of a particular technology, we mean converters developed by that technology provider to let its users communicate with users of the other technology. This distinction is moot when using symmetric converters, but worth highlighting as the model allows it.

\section{A. Impact on Adoption Levels}

We begin our investigation with a simple numerical example that illustrates how converters can induce drastic changes in the adoption of network technologies. Specifically, consider the scenario of Figure 3 that shows two adoption outcomes for the same two network technologies $\left(p_{1}=1.01, q_{1}=0.7, p_{2}=\right.$ $2.5, q_{2}=2.51, \beta=3$ ), with and without converters.

The plot on the left corresponds to a scenario without converters $\left(\alpha_{1}=\alpha_{2}=0\right)$ and in which Technology 2 eventually eliminates Technology 1 and achieves full market penetration ${ }^{8}$. This corresponds to a single, stable equilibrium $(0,1)$. The right hand plot shows how the use of perfect converters results in the elimination of the original $(0,1)$ equilibrium, so that the only possible outcome of technology adoption is now one where both technologies co-exist.

Figure 3 answers our question regarding a technology's ability to avoid elimination through the introduction of converters, and thus leading to a new equilibrium adoption outcome. We now state it more formally in the following proposition.

Proposition 3: Converters can help a technology alter market equilibrium from a scenario where it has been eliminated to one where it coexists with the other technology, or even succeeds in nearly eliminating it.

The proofs of Proposition 3 and subsequent propositions can all be found in [15, Appendix D].

As discussed above, Figure 3 provides a sample configuration illustrating Proposition 3, i.e., Technology 1 goes from elimination to dominating Technology 2 simply by introducing an efficient converter. Table IV (see Appendix) identifies that the equilibrium $(0,1)$ becomes invalid when the converter efficiency of Technology 1 verifies $\alpha_{1}>1-\frac{p_{2}-p_{1}}{\beta}$. Note that since $0 \leq \alpha_{1} \leq 1$, this requires $p_{1}<p_{2}$. Assuming this is the case, the difference between the maximum intra-network benefits of Technology 2 and the maximum cross-networks (through the converter) benefits that the users of Technology 1 derive, becomes equal to the price differential between the two technologies. As a result, low-end users (with small $\theta$ values) become indifferent to choosing either technology i.e., $\theta_{2}^{1}=0$, and any further increase in $\alpha_{1}$ leads them to switching to Technology 1 . Depending on the values of the other system parameters, it is possible that further increases in $\alpha_{1}$ can allow it to nearly eliminate Technology 2. Note that while Technology 1 may succeed in nearly eliminating Technology 2, a small number of users of Technology 2 must remain present to contribute externality benefits to the users of Technology 1 . Note also that Figure 3 considers symmetric converters and thus the outcome is not one that can be changed by the other technology deploying its own converters. This is a general phenomenon, and most if not all of the results in this section also hold under the constraint of symmetric converters (we will explicitly highlight those that don't).

A similar set of results hold for Technology 2 that, under some conditions, can enjoy the same benefits from converters. The symmetric condition that allows Technology 2 to overcome elimination $((1,0)$ is now the initial equilibrium), is to introduce a converter whose efficiency $\alpha_{2}$ exceeds $\alpha_{2} \geq 1+\left(p_{2}-p_{1}\right)-\left(q_{2}-q_{1}\right)$. In other words, Technology 2 needs to develop a converter whose efficiency compensates for both the maximum intra-network benefits of Technology 1 and the difference between the price and quality differentials of the two technologies ${ }^{9}$. At that point, $\theta_{2}^{1}=1$ so that with any further improvement in its converter efficiency, Technology

\footnotetext{
${ }^{8}$ Note that this is a scenario in which Technology 1 is marginally competitive, i.e., if left alone it would achieve a relatively low market penetration.

${ }^{9}$ The price differential must be lower than the quality differential, i.e., $p_{2}-$ $p_{1}<q_{2}-q_{1}$, for this to be possible.
} 

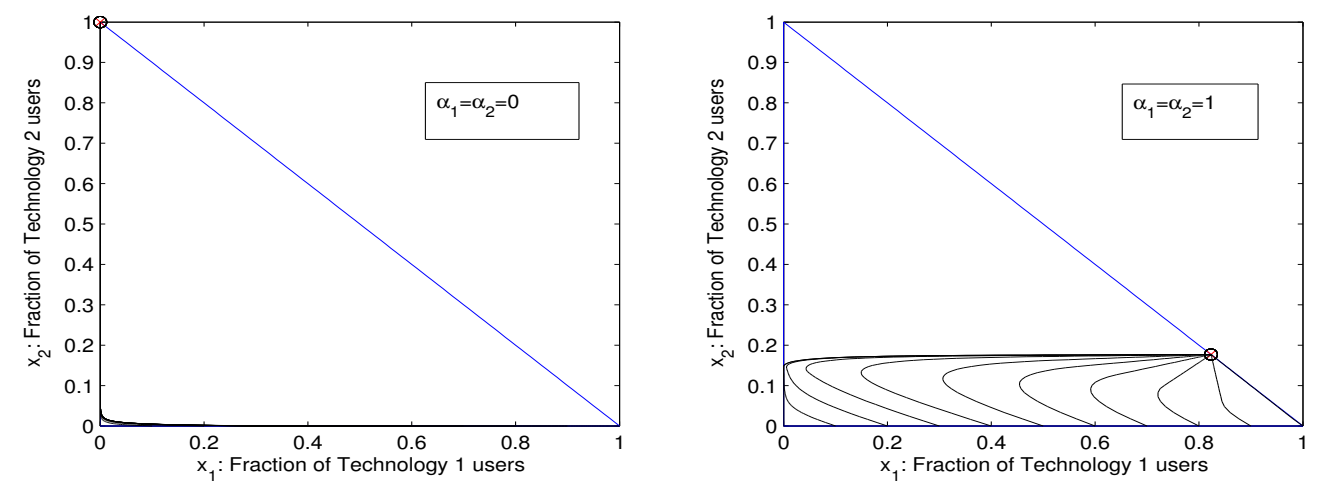

Fig. 3. On the effect of converters on the existence of equilibria. $\left(p_{1}=1.01, q_{1}=0.7, p_{2}=2.5, q_{2}=2.51, \beta=3\right)$

2 will start attracting some high-end users (large $\theta$ values) and eventually re-emerge. As with Technology 1, further improvements in its converter efficiency can in some cases allow Technology 2 to nearly wipe out Technology 1, although again not entirely.

Similar results can also be obtained from Table IV for $\left(x_{1}^{*}, 0\right)$ and $\left(0, x_{2}^{*}\right)$, i.e., instances when the elimination of a technology does not coincide with full market penetration for the other.

The behavior highlighted by Proposition 3 is relatively common. Consider our earlier example of IPv4 and IPv6. The large IPv4 installed base (or conversely, the small amount of content natively accessible over IPv6) mandates converters (gateways) that allow IPv6-only users ${ }^{10}$ to access IPv4 content. Without such converters, IPv6 is unlikely to ever take-off. Conversely, once such converters are in place, it is possible for IPv6 to eventually fully replace IPv4.

Proposition 3 focused on a scenario where converters help a technology avoid elimination. Next, we explore whether it is possible for an increase in converter efficiency to actually harm a technology, i.e., lower its market penetration.

Proposition 4: Technology 1 can hurt its market penetration by introducing a converter and/or improving its efficiency if Technology 2 offers higher externality benefits $(\beta>1)$ and the users of Technology 1 are able to access these benefits $\left(\alpha_{1} \beta>\right.$ 1). Furthermore, whenever Technology 1 hurts its own market penetration, it also reduces the overall market penetration. In contrast, Technology 2 can never hurt itself while improving its own converter efficiency.

Note that the proposition implicitly assumes asymmetric converters, i.e., explores the effect of unidirectional converter introduction or improvement.

The following discussion tries to shed light on when and why the outcome of Proposition 4 arises. Intuitively, the original impetus for Technology 1 to improve the efficiency of its converters, is to make itself more attractive to potential users by allowing them to better tap into the (higher) externality benefits of Technology 2. It may then attract new users, either from among those that had not previously adopted any

\footnotetext{
${ }^{10}$ Those that have only an IPv6 address once IPv4 addresses have been exhausted.
}

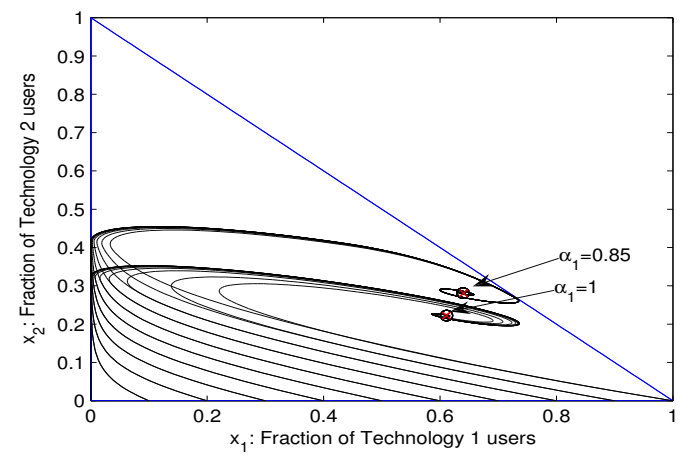

Fig. 4. Better converters harm Technology 1 and the overall market when $\alpha_{1}$ is increased from 0.85 to 1 .

$\left(p_{1}=1.3, q_{1}=0.8, p_{2}=2.3, q_{2}=2.4, \alpha_{2}=0.6, \beta=2.5\right)$

technology or among users of Technology 2 who decide to switch to Technology 1. It is the acquisition of the latter type of users that can prove harmful to Technology 1. Specifically, because $\alpha_{1} \beta>1$, the switching of users from Technology 2 to Technology 1 negatively affects the externality benefits of all Technology 1 users. When $\beta$ is high, the decrease in externality benefit can be significant. As illustrated in Figure 5, the result of this decrease can be that some low-end (small $\theta$ ) users decide to leave Technology 1 and exit the market. When the influx of new users is less than the outflow, the overall penetration of Technology 1 decreases. Figure 4 shows an instance of such a decrease. Additionally, the same reasoning shows that this also results in a decrease in overall market penetration (both $x_{1}$ and $x_{2}$ decrease).

This behavior can arise in the earlier example of competing $\mathrm{HD}$ and SD video services, as it satisfies the requirement that $\alpha_{1} \beta>1$. Specifically, although SD users are limited to generating SD quality videos, through converters they can receive and enjoy the higher-quality of HD videos. As a result, they will be negatively affected by any move of HD users back to SD. This can in turn lead some SD users to disadopt the service altogether. Hence, lowering their own user base and the overall market penetration of both services.

When $\beta \geq 1$, it is easy to see that the argument used for Technology 1 does not hold for Technology 2, i.e., acquiring a customer from Technology 1 will never decrease the external- 


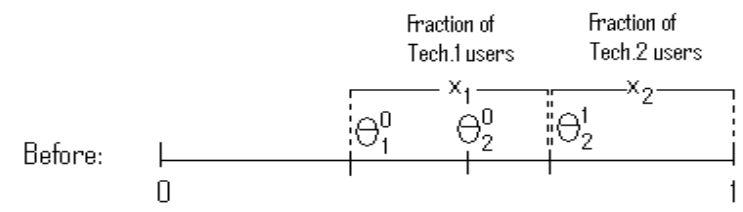

Tech. 1 improves converter efficiency

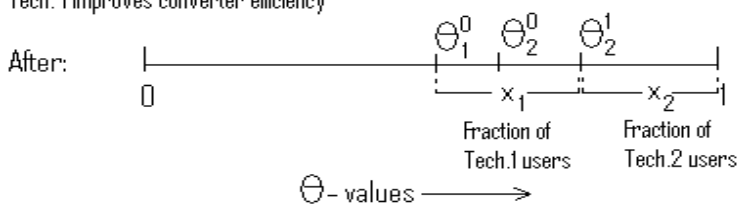

Fig. 5. Technology 1 hurts itself as well as the overall market penetration.

ity benefits of Technology 2 users, so that it cannot experience such a reversal when improving its own converter. A proof that this property actually holds for all values of $\beta$, i.e., even when $\beta \leq 1$ is provided in [15, Appendix D].

Proposition 4 indicated that Technology 1 could not only hurt itself through better converters, but also the overall market penetration. The next proposition investigates the negative impact of converters on overall market penetration, and formally identifies conditions under which this takes place.

Proposition 5: Both technologies can hurt overall market penetration through better converters. Technology 2 can have such an effect only when $\alpha_{1} \beta<1$, i.e., Technology 1 users derive lower externality benefits from connecting to Technology 2 users than to their peers. Conversely, Technology 1 demonstrates this behavior only when $\alpha_{1} \beta>1$, i.e., its users derive greater externality benefits from connecting to Technology 2 users than to their peers.

As the discussion of Proposition 4 already highlighted how this could occur with Technology 1, we focus instead on Technology 2. The motivation for better converters remains the same, namely, allow users of Technology 2 to derive higher externality benefits by connecting to users of Technology 1 . This improvement in the externality benefits of Technology 2 leads some users (those close to the $\theta_{2}^{1}$ boundary) to switch. When $\alpha_{1} \beta<1$, the migration of those users from Technology 1 to Technology 2 translates into a net drop in the overall utility Technology 1 offers its remaining users (the externality benefits contributed by every user that migrates goes down from a relative weight of 1 to one of $\alpha_{1} \beta<1$ ). This decrease in Technology 1 value then leads some low valuation users ( small $\theta$ ) to drop out altogether. Technology 1 fails to provide them with enough externality benefits to justify even its low cost, while Technology 2 remains too expensive for them. This brings the overall market penetration down.

It is interesting to note that the use of converters by Technology 2 can effectively force low valuation users to leave the market. This may not be desirable and suggests the possibility of policy interventions- regulations and/or market mechanisms- to offer low valuation users alternatives that allow them to stay in the market. This can include increasing the attractiveness of Technology 1 (e.g., subsidizing improvements in its converter efficiency), or by asking Technology 2 to provide a low-tier, low-cost version of its service that caters to low valuation users.

For a real world instance where the conditions of Proposition 5 could be satisfied, consider again the IPv4 vs. IPv6 scenario for which $\beta \approx 1$, and assume that IPv6 has taken off but that providers serving low valuation customers have not bothered with converting them to IPv6. If the converters that allow these legacy IPv4 users to access the now increasingly IPv6-only content are of low quality, it is possible that some of them will, if not drop their IPv4 service, at least significantly reduce their usage.

Figure 6 provides an example. In this configuration, in the absence of converters, Technology 1 had reached full market penetration. When Technology 2 introduces a converter of efficiency $\alpha_{2}=0.45$, it emerges and both technologies coexist at equilibrium, while still achieving full market penetration. If the efficiency of Technology 2 converter further improves, it still sees a rise in its own market penetration, but the overall market penetration now decreases to $\approx 55 \%$, as low valuation users drop out.

\section{B. Impact on Adoption Dynamics}

The previous sub-section explored the effect that converters can have on equilibria. In this sub-section we extend the investigation to both trajectories and equilibria. In particular, we concentrate on an unexpected effect of converters, one that can be shown not to be possible in their absence, namely, the possibility that the introduction of converters can render the process of technology adoption unstable. In the next proposition, we specify the conditions under which it can arise.

Proposition 6: The introduction of converters can create "boom and bust" cycles in the technology adoption process. This behavior arises only when Technology 2 exhibits higher externality benefits $(\beta>1)$ than Technology 1 and the users of Technology 1 are unconstrained in their ability to access these benefits $\left(\alpha_{1} \beta>1\right)$.

Conversely, the next corollary establishes that this never occurs in the absence of converters. The proofs are again in [15, Appendix D].

Corollary 4.1: In the absence of converters, technology adoption trajectories always converge to a stable equilibrium.

Before trying to offer some insight into the emergence of instabilities when converters are introduced, we offer an example to illustrate the type of outcomes that can arise.

Figure 7 provides a sample scenario of converters affecting the stability of technology adoption, and in particular introducing cycles in the adoption trajectories. The left-hand-side of the figure shows how in the absence of converters, Technology 2 displaces Technology 1 and achieves full market penetration. The introduction of a reasonably efficient converter $\left(\alpha_{1} \approx\right.$ 0.623 ) by Technology 1 , however, drastically changes the situation by introducing two new equilibria; both of them unstable (middle diagram). As a result, while the original equilibrium of $(0,1)$ remains valid, its basin of attraction has now shrunk considerably . Instead, under most initial conditions, a cyclical 

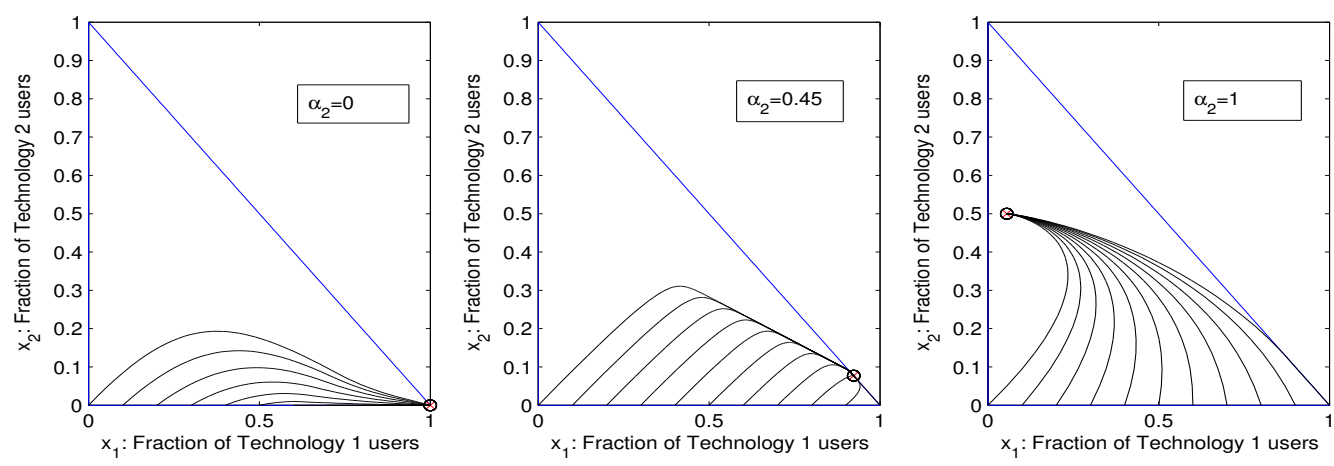

Fig. 6. Greedy Technology 2 harms overall market penetration. $\left(p_{1}=0.9, q_{1}=1.9, p_{2}=2.7, q_{2}=4.3, \alpha_{1}=0, \beta=1.2\right)$

pattern of adoption decisions emerges. In other words, users repeatedly switch back and forth between the two network technologies. Matters only become worse if the efficiency of the converter of Technology 1 continues improving ${ }^{11}$, and with a perfect converter the original equilibrium of $(0,1)$ has all but disappeared and only one, unstable equilibrium remains around which adoption decisions keep circling.

The intuition behind the emergence of such a situation is somewhat similar to that of a technology harming itself and/or the overall market through the introduction of better converters. Specifically, consider an instance where Technology 2 offers higher externality benefits that users of Technology 1 can tap into if a converter is available. When converters are absent, users that value the higher quality of Technology 2 adopt it (when it offers a higher overall utility), eventually leading to full adoption as shown on the left most part of Figure 7. However, once a converter is introduced, users have the option to remain with Technology 1 (and enjoy its lower price) without forfeiting all the benefits of Technology 2, and in particular its externality benefits. As a result, while Technology 2 will initially still gain market share by attracting high technology valuation users away from Technology 1, this now happens with Technology 1 also gaining new customers (low technology valuation customers are now adopting because of the externality benefits accessible through the new users who joined Technology 2). This combined effects results in a steady increase in overall market share until a limit is reached. This limit corresponds to a point where Technology 2 has tapped out all the high technology valuation users it could attract. As Technology 2 growth tapers off, Technology 1 continues growing as it still attracts new low technology valuation customers. Continued growth in Technology 1 customer base eventually makes it attractive to some mid-range technology valuation customers that start switching back to it. This fuels an accelerated growth in the user base of Technology 1 that now acquires customers from both Technology 2 and nonadopters. This continues until the user base of Technology 2 becomes so small that it starts affecting the ability of Technology 1 to grow. At this point, both technologies start loosing customers. This ends when the customer base of Technology 1 is small enough to allow Technology 2 to again

\footnotetext{
${ }^{11}$ As mentioned before, similar situations arise under symmetric converters.
}

start attracting customers (its own customer base had by then all but disappeared), and the process repeats anew.

To illustrate this behavior in a less abstract setting, we return to the example of HD and SD video conference services of Section II. The higher quality HD service when introduced attracts high-valuation users, who switch over from the existing SD service. This eventually results in a new market equilibrium. If the SD service responds to this competition by introducing its own converter, it will entice some (lowvaluation) non-adopters to adopt, as they now have access to the higher benefits of viewing other users in HD quality. As the number of SD users grows, the technology attracts back some of the lesser valuation HD adopters, because of its lower cost and increased externality benefits (from its larger user base and access to HD users). This results in an increase in SD adoption level and a corresponding drop in HD's. However, as the switching from HD to SD continues, the drop in the number of HD users lowers the overall externality benefits available to SD users. Consequently, the lowest valuation SD users begin to disadopt. This decrease in the number of SD users, and therefore the externality benefits that the SD service affords, makes the higher valuation SD users switch back to the HD service. This creates a situation where SD adoption drops, while the HD service grows. As before, the growth of the HD service eventually draws low-valuation users (nonadopters) to the lower-priced SD service. The two services then grow until SD's user population has once again grown large enough to attract the lesser valuation HD users. At which point the cycle repeats anew.

\section{RELATED WORK}

Modeling the diffusion of new products and technologies has a long tradition in marketing. Fourt and Woodlock [8] first proposed a product diffusion model in which a fixed fraction of consumers who have not yet bought the product do so at every period; this is known as the constant hazard rate model. Bass [1] proposed an extension that additionally incorporates word-of-mouth communication between current adopters and potential adopters. A large body of work has since built on these earlier models (see [12] for an overview of this literature). Although most of the literature deals with singleproduct settings, Norton and Bass [14] study the joint diffusion of successive generations of technologies. Their model belongs 

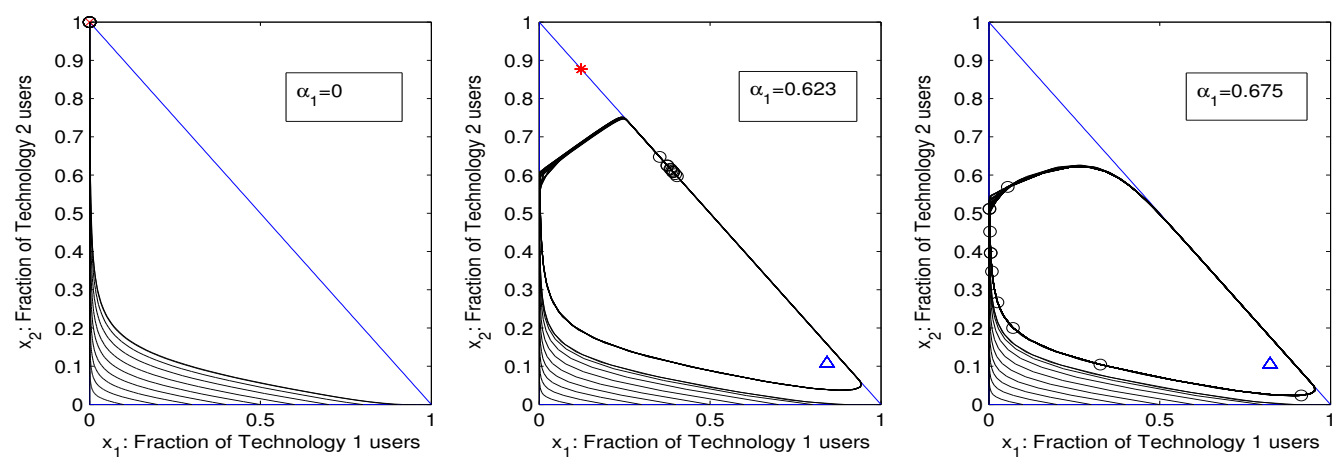

Fig. 7. Effect of converters on adoption stability $\left(p_{1}=1.05, q_{1}=0.4, p_{2}=2.1, q_{2}=2.11, \alpha_{2}=0.3, \alpha_{1}=0.675, \beta=2.8\right)$

to a class of substitution models that assume that the newer generation eventually replaces the earlier generation and thus their interest is only in the time it takes for this to occur. Significantly, both single-product and multiple-generation diffusion models focus on aggregate adoption dynamics without explicitly modeling individual decision-making processes. The advantage of such an approach is that it results in relatively simple diffusion models that can, in turn, be used to study dynamic policies (e.g., dynamic pricing). Unfortunately, these aggregate models do not shed sufficient light on the decision processes that lead to the emergent system dynamics or the exact mechanism through which various decision variables (pricing, quality, advertising, etc.) impact adoption decisions.

A few models have focused on individual-level adoption (e.g., [10]). These models provide far greater insight into the mechanism through which rational individual decision-making results in aggregate system dynamics. Given the complexity of these models, much of the progress to date has been in settings with a single technology. In contrast, the adoption of new network technologies is often influenced by incumbents. Moreover, all of the above models and indeed much of the literature refers to generic durables, e.g., washing machines. Such models do not account for the unique features of network technologies, including network externalities and the role of converters.

A recent stream of work in economics has studied the role of network externalities on equilibrium adoption of standards and technologies. Cabral [2] develops a model of individual decision-making in the presence of network externalities and characterizes the aggregate adoption dynamics. He shows that network externalities are potential drivers of S-shaped diffusion curves. We build on Cabral's model but differ in our focus by considering a two-technology setting. Put another way, we are interested in the diffusion of a new network technology in the presence of an incumbent. A related paper by Farrell and Saloner [6] evaluates the impact of an installed base on the transition to a new standard. They show that the installed base can cause "excess inertia" which prevents the transition to the new standard. At the same time, the adoption of the new standard by a few users can create "excess momentum" as well. In their model, users are homogeneous except for the time of their arrival into the system. As a result, they observe a bandwagon effect in which the adoption of a standard by one set of users makes the same choice more attractive to all other users. Thus, one standard always wins and coexistence is not feasible. Choi [3] extends the model by Farrell and Saloner [6] to include converters and shows that converters can in some instances blockade the transition by weakening the threat of being stranded for users of the incumbent technology. In a more recent study, Joseph et al. [11] also show that increase in efficiency of a converter can hinder the adoption of a new network architecture.

An important distinction of our work relative to these papers is that we incorporate heterogeneity in user preferences. We show that this gives rise to equilibria in which the technologies may coexist, i.e., neither network technology fully captures the market. Further, very little attention is paid to the adoption path in these papers because all users make the same decision. In contrast, we show that the heterogeneity across users can result in interesting adoption dynamics including non-monotonic evolution of the market shares of the technologies. Additionally, these papers focus on environments in which users make the decisions to adopt the converters. This is meaningful in environments in which the converter functionality and its deployment resides with individual users, e.g., converters for two incompatible software applications that a user decides to download. In contrast, our interest is in environments in which converters are usually deployed by the technology providers upon incurring high fixed costs, and in the process made available to all its users.

\section{Conclusion And Extensions}

The paper provides a framework to study the adoption and diffusion of a new network technology in the presence of an incumbent and offers insight into the role of converters. Our model accounts for both externalities and user heterogeneity, and helps reveal several unexpected behaviors. Of note are that the presence of converters can hurt overall market penetration, and that under certain conditions they can preclude the adoption process from ever converging. In [15, Appendix $F$ and $G$ ], we show that these behaviors remain present across a wide range of utility models that differ from the one used for analytical tractability in this paper. These robustness tests consider nonlinear externality functions, nonuniform distribution of user preferences, user heterogeneity in both standalone and network benefits and switching costs. 
As the first step of our investigation in the dynamics of technology adoption in the presence of converters, the paper and its model clearly have limitations that we plan to address in the future. As mentioned earlier, allowing some of the system parameters to be time-varying is of obvious interest. Similarly, letting prices be endogenous and/or dynamic variables that are chosen by strategic service providers is another direction we have started investigating.

Our work represents an initial step towards understanding adoption dynamics of network technologies. Further work building on this paper would likely provide additional insight.

\section{ACKNOWLEDGMENTS}

The authors would like to acknowledge Z.-L. Zhang's input to an earlier version of the work and A. Odlyzko's feedback.

\section{APPENDIX}

The expressions for equilibria in $R_{5}$ and $R_{6}, \underline{x}_{R_{5}}^{*}$ and $\underline{x}_{R_{6}}^{*}$ of Tables III, IV and V are provided separately as eqs. (12) and (13) respectively for better readability.

$$
\begin{aligned}
x_{1}^{*} R_{5} & =\frac{\left(p_{2}-p_{1}\right)-\beta\left(1-\alpha_{1}\right)}{\left(q_{2}-q_{1}\right)-\left[\left(1-\alpha_{2}\right)+\beta\left(1-\alpha_{1}\right)\right]} \\
x_{2}^{*} R_{5} & =1-x_{1}^{*} R_{5}=\frac{\left(q_{2}-q_{1}\right)-\left(p_{2}-p_{1}\right)-\left(1-\alpha_{2}\right)}{\left(q_{2}-q_{1}\right)-\left[\left(1-\alpha_{2}\right)+\beta\left(1-\alpha_{1}\right)\right]} \\
x_{1}^{*} R 6= & \frac{p_{1} q_{2}-p_{2} q_{1}+\beta \alpha_{1}\left(p_{2}-q_{2}\right)-\beta\left(p_{1}-q_{1}\right)}{\left(q_{1}-1\right)\left(\beta-q_{2}\right)+\left(q_{1}-\alpha_{1} \beta\right)\left(q_{1}-\alpha_{2}\right)} \\
x_{2}^{*} R 6= & \frac{p_{2} q_{1}-p_{1} q_{1}-p_{2}+p_{1} \alpha_{2}+q_{1}^{2}-q_{1} q_{2}+q_{2}-q_{1} \alpha_{2}}{\left(q_{1}-1\right)\left(\beta-q_{2}\right)+\left(q_{1}-\alpha_{1} \beta\right)\left(q_{1}-\alpha_{2}\right)}
\end{aligned}
$$

\section{REFERENCES}

[1] F. M. Bass. A new product growth for model consumer durables. Management Science, 15(5):215-227, 1969.

[2] L. M. B. Cabral. On the adoption of innovation with network externalities. Mathematical Social Sciences, 19:299-308, 1990.

[3] J. P. Choi. Do converters facilitate the transition to a new incompatible technology?: A dynamic analysis of converters. International Journal of Industrial Organization, 14:825-836, 1996.

[4] D. Demirhan, V. S. Jacob, and S. Raghunathan. Strategic it investments: the impact of switching cost and declining it cost. Management Science, 53(2):208-226, 2007.

[5] A. Durand, editor. Dual-stack lite broadband deployments post IPv4 exhaustion. Internet Draft (draft-ietf-softwire-dual-stack-lite-04.txt), March 2010. (Work in progress).

[6] J. Farrell and G. Saloner. Installed base and compatibility: Innovation, product preannouncements, and predation. American Economic Review, 76:940-955, 1986.

[7] J. Farrell and G. Saloner. Converters, compatibility, and the control of interfaces. The Journal of Industrial Economics, 40(1):9-35, 1992.

[8] L. A. Fourt and J. W. Woodlock. Early prediction of market success for grocery products. Journal of Marketing, 25:31-38, 1960.

[9] P. E. Green, A. Krieger, and Y. Wind. Thirty years of conjoint analysis: Reflections and prospects. Interfaces, 31(3):S56-S73, 2001.

[10] D. Horsky. A diffusion model incorporating product benefits, price, income and information. Marketing Science, 9(4):342-365, 1990.

[11] D. Joseph, N. Shetty, J. Chuang, and I. Stoica. Modeling the adoption of new network architectures. In Proc. ACM CoNEXT'07, 2007.

[12] V. Mahajan, E. Muller, and Y. Wind, editors. New-Product Diffusion Models. Springer, 2000.

[13] B. Martorelli. Internet service providers: Addressing the customer service challenge. White paper, Hurwitz Group Inc., Framingham, MA., 2000.

[14] J. A. Norton and F. M. Bass. A diffusion theory model of adoption and substitution for successive generations of high-technology products. Management Science, 33(9):1069-1086, 1987.

[15] S. Sen, Y. Jin, R. Guérin, and K. Hosanagar. Modeling the dynamics of network technology adoption and the role of converters. Technical report, University of Pennsylvania, June 2009. Available at http://repository.upenn.edu/ese_papers/496/.

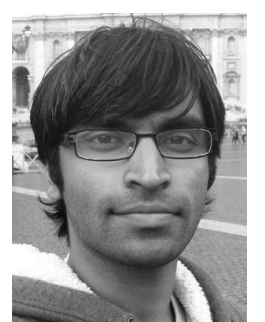

Soumya Sen received his B.E.(Hons.) in Electronics \& Instrumentation Engineering from Birla Institute of Technology and Sciences (BITS, Pilani), India, in 2005, and M.S. degree in Electrical Engineering from the University of Pennsylvania in 2007, where he is currently working toward his Ph.D. degree.

His research interests are in communication networks architectures, Internet economics, ecommerce and social networks. In August 2008, he served as the session chair for 'Network Economics and Industry Structure' at the NSF FIND Student

Workshop held in Seattle.

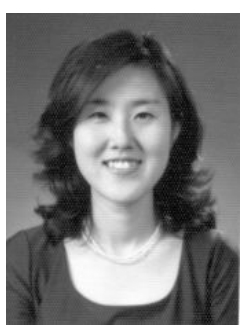

Youngmi Jin received her B.S. and M.S. degrees in Mathematics from KAIST. She was a Member of Technical Staff at Korea Telecom Research and Development Center, where she did research on traffic engineering and broadband wireless access (IEEE 802.16) system. She received her M.S. and Ph.D. degrees in Electrical Engineering from the Pennsylvania State University. Her research interests are communication networks, wireless networks, Internet economics, Internet pricing, incentive engineering and social networks.

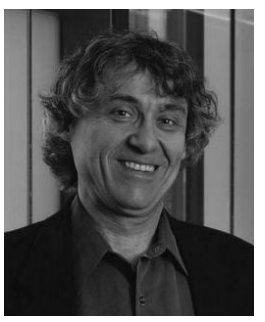

Roch Guérin (F IEEE '01 / F ACM '06) received an engineer degree from ENST, Paris, France, and M.S. and Ph.D. degrees in EE from Caltech.

From 1986 till 1998 he was with the IBM T. J. Watson Research Center, Yorktown Heights, NY. In 1998 he joined the Electrical and Systems Engineering department of the University of Pennsylvania, as the Alfred Fitler Moore Professor of Telecommunications Networks. His research has spanned core networking issues, e.g., routing, traffic engineering, quality-of-service, etc., as well as topics related to the use of networks by applications and the exploration of how economic factors affect the evolution of networked systems.

Dr. Guérin has been an editor for several ACM and IEEE publications, and has been involved in the organization of numerous ACM and IEEE sponsored activities. He was General Chair of the IEEE INFOCOM'98 conference, Technical Program co-chair of the ACM SIGCOMM'01 conference, General Chair of the ACM SIGCOMM'05 conference, and Program co-chair of the ACM CoNEXT'07 conference. He currently serves on the ACM CoNEXT steering committee. In 1994 he received an IBM Outstanding Innovation Award for his work on traffic management and the concept of equivalent bandwidth, and in 2010 he received the IEEE INFOCOM Achievement Award for "Pioneering Contributions to the Theory and Practice of QoS in Networks." He was on the Technical Advisory Board of France Telecom for two consecutive terms from 2001 to 2006, and on that of Samsung Electronics in 2003-2004.

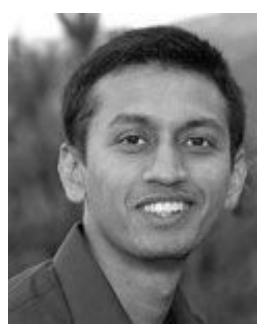

Kartik Hosanagar is an associate professor of Operations and Information Management at The Wharton School, University of Pennsylvania. His research interests are in Internet-enabled commerce, with a special emphasis on Internet media and marketing and distributed networking. He has a Bachelors degree in Electronics and a Masters in Information Systems, both from Birla Institute of Technology and Sciences (BITS, Pilani), India and an MPhil in Management Science and a PhD in Management Science and Information Systems from the Heinz School of Policy at Carnegie Mellon University. He serves as an Associate Editor of Information Systems Research. 
TABLE III

TECHNOLOGY ADOPTION TRAJECTORIES

\begin{tabular}{|c|c|c|}
\hline & $x_{1}(t)$ & $x_{2}(t)$ \\
\hline$R_{1}$ & $x_{1}\left(t_{0}\right) e^{-\gamma\left(t-t_{0}\right)}$ & $\frac{\left(x_{2}\left(t_{0}\right)-1\right)}{x_{1}\left(t_{0}\right)} e^{-\gamma t}+1$ \\
\hline \multirow[t]{2}{*}{$R_{2}$} & $c_{1} e^{-\gamma\left(t-t_{0}\right)}$ & $\frac{p_{2}-q_{2}}{\beta-q_{2}}+c_{2} e^{-\gamma\left(1-\beta / q_{2}\right)\left(t-t_{0}\right)}-c_{1} \frac{\alpha_{2}}{\beta} e^{-\gamma t}$ \\
\hline & $c_{1}=x_{1}\left(t_{0}\right)$ & $c_{2}=\left[x_{2}\left(t_{0}\right)+\frac{\alpha_{2} x_{1}\left(t_{0}\right)}{\beta}-\frac{p_{2}-q_{2}}{\beta-q_{2}}\right]$ \\
\hline$R_{3}$ & $x_{1}(t)=x_{1}\left(t_{0}\right) e^{-\gamma\left(t-t_{0}\right)}$ & $x_{2}(t)=x_{2}\left(t_{0}\right) e^{-\gamma\left(t-t_{0}\right)}$ \\
\hline$R_{4}$ & same as $R_{1}$ & same as $R_{1}$ \\
\hline \multirow[t]{2}{*}{$R_{5}$} & $x_{1 R_{5}}^{*}+c_{2} e^{-\gamma\left(t-t_{0}\right)}+c_{1} e^{\left(-1+\frac{\left(1-\alpha_{2}\right)+\beta\left(1-\alpha_{1}\right)}{q_{2}-q_{1}}\right) \gamma\left(t-t_{0}\right)}$ & $x_{2}^{*} R_{5}+c_{2} \frac{\left(1-\alpha_{2}\right)}{\beta\left(1-\alpha_{1}\right)} e^{-\gamma\left(t-t_{0}\right)}-c_{1} e^{\left(-1+\frac{\left(1-\alpha_{2}\right)+\beta\left(1-\alpha_{1}\right)}{q_{2}-q_{1}}\right) \gamma\left(t-t_{0}\right)}$ \\
\hline & $\begin{aligned} c_{1} & =\frac{\beta\left(1-\alpha_{1}\right)}{1-\alpha_{2}+\beta\left(1-\alpha_{1}\right)}\left(x_{2}^{*} R_{5}-x_{2}\left(t_{0}\right)\right) \\
& -\frac{1-\alpha_{2}}{1-\alpha_{2}+\beta\left(1-\alpha_{1}\right)}\left(x_{R_{5}}^{*}-x_{1}\left(t_{0}\right)\right)\end{aligned}$ & $c_{2}=\left(\frac{\beta\left(1-\alpha_{1}\right)}{1-\alpha_{2}+\beta\left(1-\alpha_{1}\right)}\right)\left[x_{1}\left(t_{0}\right)+x_{2}\left(t_{0}\right)-1\right]$ \\
\hline \multirow[t]{2}{*}{$R_{6}$} & $x_{1 R_{6}}^{*}+c_{1} K_{1} e^{\frac{A+\sqrt{A^{2}-4 B}}{2} \gamma\left(t-t_{0}\right)}+c_{2} K_{2} e^{\frac{A-\sqrt{A^{2}-4 B}}{2} \gamma\left(t-t_{0}\right)}$ & 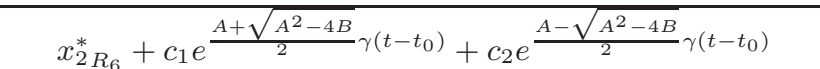 \\
\hline & $\begin{array}{c}c_{1}=\frac{\left(1-\alpha_{2}\right)\left\{x_{1}^{*} R_{6}-x_{1}\left(t_{0}\right)-K_{2}\left(x_{2}^{*} R_{6}-x_{2}\left(t_{0}\right)\right)\right\}}{\left(q_{2}-q_{1}\right) \sqrt{A^{2}-4 B}} \\
K_{1}=\frac{\alpha_{2}+\beta\left(1-\alpha_{1}\right)-q_{2} / q_{1}-\left(q_{2}-q_{1}\right) \sqrt{A^{2}-4 B}}{2\left(1-\alpha_{2}\right)} \\
K_{2}=\frac{\alpha_{2}+\beta\left(1-\alpha_{1}\right)-q_{2} / q_{1}+\left(q_{2}-q_{1}\right) \sqrt{A^{2}-4 B}}{2\left(1-\alpha_{2}\right)}\end{array}$ & $\begin{array}{c}c_{2}=\frac{\left(1-\alpha_{2}\right)\left\{-\left(x_{1}^{*} R_{6}-x_{1}\left(t_{0}\right)\right)+K_{1}\left(x_{2}^{*} R_{6}-x_{2}\left(t_{0}\right)\right)\right\}}{\left(q_{2}-q_{1}\right) \sqrt{A^{2}-4 B}} \\
A=\frac{1-\alpha_{2}+\beta\left(1-\alpha_{1}\right)}{q_{2}-q_{1}}+\frac{1}{q_{1}}-2 \\
B=\left(\frac{1}{q_{1}}-1\right)\left(\frac{\beta\left(1-\alpha_{1}\right)}{q_{2}-q_{1}}-1\right)+\frac{1-\alpha_{2}}{q_{2}-q_{1}}\left(\frac{\beta \alpha_{1}}{q_{1}}-1\right)\end{array}$ \\
\hline$R_{7}$ & $\left(x_{1}\left(t_{0}\right)-1\right) e^{-\gamma\left(t-t_{0}\right)}+1$ & $x_{2}\left(t_{0}\right) e^{-\gamma\left(t-t_{0}\right)}$ \\
\hline$R_{8}$ & $\begin{array}{c}\frac{p_{1}-q_{1}}{1-q_{1}}+c_{1} e^{-\gamma\left(1-\frac{1}{q_{1}}\right)\left(t-t_{0}\right)}-c_{2} \beta \alpha_{1} e^{-\gamma\left(t-t_{0}\right)} \\
c_{1}=\left[x_{1}\left(t_{0}\right)+\beta \alpha_{1} x_{2}\left(t_{0}\right)-\frac{p_{1}-q_{1}}{1-q_{1}}\right]\end{array}$ & $\begin{array}{l}c_{2} e^{-\gamma\left(t-t_{0}\right)} \\
c_{2}=x_{2}\left(t_{0}\right)\end{array}$ \\
\hline$R_{9}$ & $x_{1}(t)=x_{1}\left(t_{0}\right) e^{-\gamma\left(t-t_{0}\right)}$ & $x_{2}(t)=x_{2}\left(t_{0}\right) e^{-\gamma\left(t-t_{0}\right)}$ \\
\hline
\end{tabular}

TABLE IV

CONDITIONS FOR STABLE, VALID EQUILIBRIA

\begin{tabular}{|c|c|c|c|}
\hline Region & Equilibria & Stability Conditions & Validity and Stability Conditions \\
\hline$R_{1}$ & $(0,1)$ & always locally stable & $p_{2} \leq \beta, \alpha_{1} \leq \frac{p_{1}}{\beta}+\frac{q_{1}}{q_{2}}\left(1-\frac{p_{2}}{\beta}\right)$ \\
\hline$R_{2}$ & $\left(0, \frac{p_{2}-q_{2}}{\beta-q_{2}}\right)$ & $\beta<q_{2}$ & $\beta<p_{2}<q_{2}$ \\
& & & $\alpha_{1} \beta\left(q_{2}-p_{2}\right) \leq \beta\left(q_{1}-p_{1}\right)+p_{1} q_{2}-p_{2} q_{1}$ \\
\hline$R_{4}$ & $(0,1)$ & always locally stable & $p_{1}<\alpha_{1} \beta, \frac{p_{1}}{\beta}+\frac{q_{1}}{q_{2}}\left(1-\frac{p_{2}}{\beta}\right) \leq \alpha_{1} \leq 1+\frac{p_{1}-p_{2}}{\beta}$ \\
\hline$R_{5}$ & $\left(x_{1 R_{5}}^{*}, 1-x_{1}^{*} R_{5}\right)$ & $q_{2}-p_{1}>1-\alpha_{2}+\beta\left(1-\alpha_{1}\right)$ & $p_{2}-q_{1}-\left(p_{2}-p_{1}\right) \geq 1-\alpha_{2}$ \\
& $($ See Eq. $(12))$ & & $q_{2}-q_{1}>\beta\left(1-\alpha_{1}\right)+1-\alpha_{2}$ \\
& & & $\alpha_{1} \beta\left(\alpha_{2}+q_{2}-q_{1}-p_{2}\right) \geq \beta-p_{2}-p_{1}\left(\beta-\alpha_{2}-\left(q_{2}-q_{1}\right)\right)$ \\
\hline$R_{6}$ & $\left(x_{\left.1 R_{6}, x_{2}^{*} R_{6}\right)}^{*}\right.$ & See Table V & $0<x_{1}^{*} R_{6}, 0<x_{2}^{*}, 0<x_{1}^{*}+x_{2}^{*} R_{6}<1$ \\
\hline$R_{7}$ & $($ See Eq. $(13))$ & $p_{1} \leq 1, \alpha_{2} \leq 1+p_{2}-p_{1}-\left(q_{2}-q_{1}\right)$ \\
\hline$R_{8}$ & $(1,0)$ & always locally stable & $1<p_{1}<q_{1}$ \\
& $\left(\frac{p_{1}-q_{1}}{1-q_{1}}, 0\right)$ & $1<q_{1}$ & $\alpha_{2}\left(q_{1}-p_{1}\right) \leq\left(1-q_{1}\right)\left(q_{2}-p_{2}\right)+q_{1}\left(q_{1}-p_{1}\right)$ \\
\hline
\end{tabular}

TABLE V

StABILITy CONDITIONS FOR $\underline{x}_{R_{6}}^{*}$

\begin{tabular}{|c|c|}
\hline Case & Conditions \\
\hline \hline$A^{2}-4 B \geq 0$ & $A<0 \Leftrightarrow \beta\left(1-\alpha_{1}\right)-\alpha_{2}<2\left(q_{2}-q_{1}\right)-\frac{q_{2}}{q_{1}}$ \\
(Ref. Table III for exp. of A and B) & $B>0 \Leftrightarrow\left(q_{1}-1\right)\left(\beta-q_{2}\right)+\left(q_{1}-\alpha_{1} \beta\right)\left(q_{1}-\alpha_{2}\right)<0$ \\
\hline \hline$A^{2}-4 B<0$ & $A<0 \Leftrightarrow \beta\left(1-\alpha_{1}\right)-\alpha_{2}<2\left(q_{2}-q_{1}\right)-\frac{q_{2}}{q_{1}}$ \\
\hline
\end{tabular}

